\title{
Anomaly Detection in NFV Using Tree-Based Unsupervised Learning Method
}

\author{
Girish $L^{a}$, Dr. Sridhar K N Rao*b \\ ${ }^{a}$ Research Scholar, Department of CSE, CIT Gubbi, Karnataka, India; \\ ${ }^{b}$ Professor, Department of CSE, CIT Gubbi, Karnataka, India;
}

A R T I C L E H I S T O RY

Received: 2019-06-19

Revised: 2019-07-25

Accepted: 2019-07-25
Abstract: With the increased adoption of virtualized NFs in data center, it is crucial to address some of the challenges such as performance and availability of the applications in virtualized network environment. The normal operation of the network can be analyzed with respect to the usage of various resources like, CPU, memory, network and disk. Inefficient usage or over usage of these resources leads to anomalous behavior. Anomalies are often preceded by faults. It is important to detect anomalies before they occur. The detected anomalies can be used for corrective and optimization actions. This paper presents that; unsupervised machine learning algorithm performs better compared to supervised machine learning algorithms in detecting anomalies. Here we use isolation forest algorithm on time series dataset, which is collected using monitoring agent collectd. Stress is induced to the computer using traffic monitoring generator and stress-ng. The results show that isolation forest algorithm gives better performance in anomaly detection with good anomaly score.

Keywords: SDN, NFV, Anomaly, Machine Learning, Random Forest. Openstack.

\section{INTRODUCTION}

NFV and SDN are emerging paradigms in networking, where the network functions (NFs) are migrated from traditional hardware based devices to software based virtual applications. The rapid adoption of SDN and NFV in telecom industry is experiencing an ever-increasing attention both from academia and network community as they reduce the CAPEX and OPEX by introducing virtual capabilities in the network infrastructure. [2]

However, there are a few challenges to be handled with the adoption of virtualized NFs. One of those challenges is performance and availability of the applications once they are migrated into virtualized environment. In order to address these challenges, an integrated analytical monitoring system is needed, which collects the metrics from both virtualized applications and physical infrastructure.

*Research Scholar, Department of CSE, Channabasaveshwara Institute of Technology, E-mails: girish.1@ cittumkur.org
In traditional monitoring approaches, active and passive monitoring techniques like status and heartbeats requests are used to identify the anomaly. These techniques detect the anomaly only after they happen. Identifying and correcting the anomalies before their occurrence will improve the performance of the system.

Today, the monitoring systems are able to produce alarms with threshold that is already pre-defined based on the earlier Knowledge of the network. Where as in case of large-scale NFV environment, having dynamic network, managing such static thresholds for each VNFs are not efficient. [20]

Therefore, it is required to build an automated system to identify the anomalies in NFV environments without using predefined threshold. Such an automated system can detect as well as predict the faults in time. Artificial intelligence and machine learning algorithms learns from historical data to detect and predict the anomalies efficiently. [19][21].

In network, different types of anomalies exhibits themselves in different manner, therefore, it is difficult to build the training set with labels as normal network behavior and abnormal network behavior (anomalies). These data sets are usually not available, and they can be difficult to collect and maintain. Hence, supervised model based algorithms are not suitable in the dynamic nature of the network data for anomaly detection. [1] 
Unsupervised anomaly detection methods are the most flexible methods, as they do not require labels for the dataset. There exists no difference between the training set and the test set in case of unsupervised methods. This is because, unsupervised anomaly detection methods scores the data considering the intrinsic properties of the dataset. Unsupervised methods assume that only a small fraction of the dataset show significantly different behavior compared to the normal data.

The focus in this paper is to work on assessing the efficiency of a tree based unsupervised anomaly detection approach applied on a NFV virtual environment. In the proposed tree structure, every single instance of data point can be isolated effectively. The high susceptible nature of isolation forest helps in isolating anomalies towards the root of the tree and normal data points towards the other end of the tree. The capability of isolating the anomalies from normal points is the fundamental characteristic that helps in detecting the anomalies. Such a type of tree is called Isolation Tree or iTree.

The rest of the paper is organized as follows, section II briefs about the types of anomalies and the related work. Section III describes about the approach used. Experimental details are discussed in Section IV followed by the conclusion.

\section{CATEGORIZATION OF ANOMALY DETECTION}

Anomaly detection is a technique of identifying the data items that do not exhibit expected behavior. The presence of such anomalous data patterns in the network traffic results in problems such as structural defects, errors or frauds. Detecting the anomalous data patterns will provide additional information to the network administrator, which helps in identifying the network behavior and discovering the root cause of faults in a network.

\subsection{Types of Anomaly}

There are three types of anomalies, point anomalies, contextual anomalies and collective anomalies. Point anomalies are the easiest type of anomalies. They are the observations made in which a single instance is anomalous when compared to all remaining instances. Contextual Anomalies also called conditional anomalies are based on particular context commonly observed in time series data. Observation made is unusual in certain context but not in other. Collective Anomalies are those in which a set of data instances are anomalous with respect to the whole data set. This type of anomaly considers relationship among data instances. The individual instances are not anomalous by themselves.

\subsection{Anomaly Detection Techniques}

Most of the methods of network anomaly detection are based on network traffic models. In case of cloud networks, various techniques have been used for anomaly detection such as threshold detection, statistical analysis, data mining and machine learning. This section presents a comparative study of various methods used for anomaly detection in cloud networks.
1) Statistical based methods: Statistical based methods assume that normal data are associated with high probability states where as anomalous data are associated with low probability states of the process.

M A Kourtis et al in [3] have used statistical methods like root mean square error [4] and covariance matrix [5] for detecting the anomalies in network function virtualization services. They perform the anomaly detection on system matrices (CPU load and memory utilization) using multiple linear regression and mahalanobis distance. The performance of both methods exhibits similar results. In statistical based approaches, it is difficult to estimate distributions in high dimensional data.

2) Machine Learning Based Anomaly Detection: In cloud environment, anomaly detection is done using statistical and machine learning techniques. This section presents an overview of recent work on anomaly detection in NFV environment.

In case of classification techniques, various normal data instances are learned by the classifiers. Data instance that is not classified as normal by any of the classifiers is considered as abnormal.

Anton Gulenko et al in [6] conducted experiments for anomaly detection in cloud environment. They used supervised algorithms such as random forest, SVM and decision tree.

Evaluated the results of each algorithm based on their precision, recall and F1 score. Experiments were conducted using the project clearwater as virtualized IP-multimedia system based on the consequences like memory leak, high CPU consumption, RAM utilization and disk IO.

Carla Sauvanaud et al in [7] used tree-based algorithm, random forests for anomaly detection on VNF monitoring data. They presented results evaluating receiver operating characteristics (ROC) and the precision call (PR).

MingXue Wang et al in [8] performed anomaly detection on streaming data for evaluating network management system.

Panpan Zheng et al [9] used semi-supervised method, Oneclass SVM for anomaly detection. One-class SVM is one of the most widely used semi-supervised learning technique. It gives good results even for high dimensional datasets.

Masanori Miyazawa et al in [10] [11] carried out experiments for anomaly detection in NFV environment using unsupervised learning technique, self-organizing maps (SOM).

It is a form of neural network. SOM can analyze different types of data even using small amount of computer resources. It is based on decentralized approach. The models for each application were built using hypervisor level information and VM level information [13].

Solaimani et al [14] tried a different anomaly detection approach, which is capable of handling VM monitoring data as streams. This approach is based on clustering technique. VM monitoring data was grouped into different clusters and the machines that do not belong to any clusters are considered as outliers or anomalous. 


\section{APPROACH}

There are various approaches available for anomaly detection; [1] unsupervised learning approaches have given promising performance in detecting anomalies. In particular, isolation forest algorithm has made a mark in the literature for its efficiency and good accuracy in detecting anomalies in NFV environment. In this paper, we present an evaluation conducted on the results of Isolation forest algorithm.

\subsection{Isolation Forest}

Isolation forest works based on decision tree. It creates a bunch of decision trees. It can be created in such a way that it is possible to isolate every single instance. Isolation forests are mainly used for outlier detection and anomaly detection. As the name indicates, isolation forest separates one data instance from rest of the data instances. Anomalous data points are kept closer to the depth of the tree and normal data point towards the root. Isolation forest randomly selects the feature and randomly splits the maximum and minimum values of the feature.

The Fig 1 shown below is a data induced random tree. The instances are partitioned repeatedly and recursively until every instance gets isolated. This method produces shorter paths for anomalies. Small number of anomaly instances result in smaller number of partitions i.e. shorter paths for anomalies.

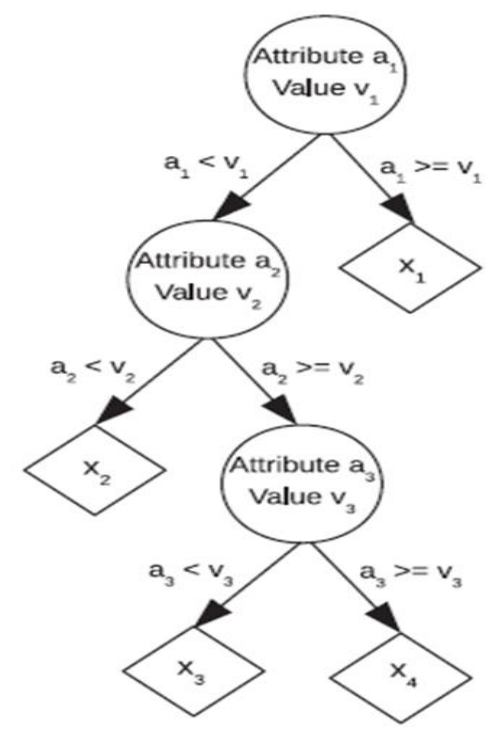

Fig. 1. Overview of an Isolation tree.

\subsubsection{Scaling/Resolution}

Instances having distinguishable attribute-values are expected to be partitioned in early partitioning. Thus if a forest of random trees produces shorter paths for particular instances, then such instances can be highly suspected to be anomalies.

Anomaly detection using isolation forest consists of two steps. In the first stage, isolation trees are constructed and the given training instances are recursively partitioned until all the instances are isolated. It uses subsample size to control the training data size. In the second stage, the test instances are passed through isolation trees and the anomaly score for each instance is obtained.

$$
S(x, n)=2-(E(h(x))) /(c(n))
$$

Where $\mathrm{h}(\mathrm{x})$ is path length of observation $\mathrm{x}, \mathrm{c}(\mathrm{n})$ is average path length of unsuccessful in a binary search trees, $\mathrm{N}$ is the number of external nodes.

Every instance is assigned with anomaly score and the following inferences are drawn based on the anomaly score:

- The instances having anomaly score nearer to one are considered as anomalous.

- The instances having anomaly score smaller than 0.5 are considered as normal instances.

- If the anomaly score is closer to 0.5 , it indicates the entire dataset does not consist of anomalies.

Isolation forests give good results for huge data sets with several dimensions. It does not rely on distance and density measures for anomaly detection. It has a linear time complexity with low memory management.

\subsection{Experimental Setup}

The experimental setup is shown in the Fig 2, where the monitoring data metrics is collected in the form of time series data using monitoring agent collectd [16]. Collectd collects the metrics from various data sources like the operating system, application and cloud infrastructure. It sends the collected information over the network and stores the information in a time series database InfluxDB [15]. The collected dataset is sampled periodically at the time interval of 1 second.

InfluxDB is similar to structured query language, which consists of predefined functions to query the data points. Each data point is composed of key-value pair where key is a field set and value is timestamp. InfluxDB supports visualization tool grafana and acts as a data source.

The anomalies are injected artificially to create abnormal behavior in the generated dataset. The abnormal behavior can occur because of high cpu consumption, memory leak and huge packet loss.

To inject anomalies artificially we use traffic generator iperf [18] by sending millions of packets at specific time, this leads to packet loss and increased latency. In addition, we induce more stress on the computer resources like CPU, memory and disk using stress-ng tool [17]. 


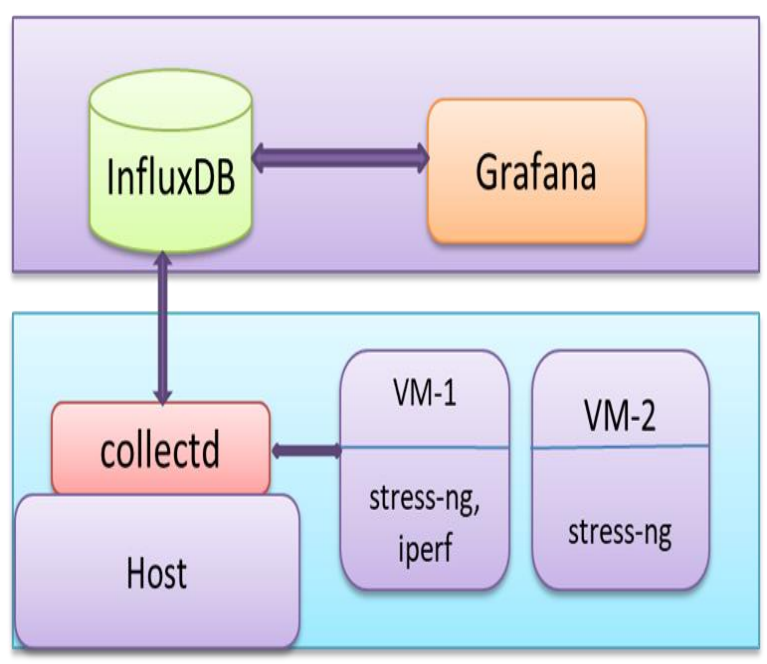

Fig. 2. Experimental Setup

\section{EVAUATION AND RESULTS}

To evaluate the accuracy of isolation forest algorithm in detecting anomalies, we used anomaly injection and detection method. The data for the experiment was collected from two virtual machines and one host machine for the duration of 24 hours at the interval of one second. The collected dataset consists of 12 features (metrics). The anomalies are injected randomly for the duration of 6 to 8 minutes.

Table I gives the details of the different metrics considered for the experiment. Fig (3) and (4) shows the evaluation of anomaly detection using isolation forest. The anomaly score were calculated with different types of workload and the distribution of the anomaly path length is plotted using histogram and boxplots. We see that, there is a clear cluster under -0.05 . We consider average path lengths shorter than 0.05 as anomalies.

Table 1: System Metrics Used

\begin{tabular}{ll}
\hline Metric & Description \\
CPU & CPU utilization \\
Ram & $\begin{array}{l}\text { Ram Utilization } \\
\text { Performance statistics of hard- } \\
\text { disks }\end{array}$ \\
Disk IO & Hard disk access in bytes \\
Network I O & Statistics about processes \\
Number of processes & $\begin{array}{l}\text { Utilization of a machine in } \\
\text { percentage }\end{array}$
\end{tabular}


Software Defined Networks (NFV-SDN), Palo Alto, CA, 2016, pp. 161-166.

[4] Root Mean Square Error. https://en.wikipedia.org/wiki/Root-meansquare- deviation [5]Covariancematrix. https://en.wikipedia.org/wiki/Covariance-matrix

[6] A. Gulenko, M. Wallschlger, F. Schmidt, O. Kao and F. Liu, "Evaluating machine learning algorithms for anomaly detection in clouds" IEEE International Conference on Big Data (Big Data), Washington, DC, 2016, pp. 2716-2721.

[7] C. Sauvanaud, K. Lazri, M. Kaniche and K. Kanoun, "Towards Black-Box Anomaly Detection in Virtual Network Functions" 46th Annual IEEE/IFIP International Conference on Dependable Systems and Networks Workshop (DSN-W), Toulouse, 2016, pp. 254-257.

[8] M. Wang and S. Handurukande, "A Streaming Data Anomaly Detection Analytic Engine for Mobile Network Management", Intl IEEE Conferences on(UIC/ATC/ScalCom/CBDCom/IoP/SmartWorld), Toulouse, 2016, pp. 722-729.

[9] Y. Wang, J. Wong and A. Miner, "Anomaly intrusion detection using one class SVM", Proceedings from the Fifth Annual IEEE SMC Information Assurance Workshop, 2004, pp. 358-364. doi: 10.1109/IAW.2004.1437839.

[10] M. Miyazawa, M. Hayashi and R. Stadler, "vNMF:Distributed fault detection using clustering approach for network function virtualization" IFIP/IEEE International Symposium on Integrated Network Management (IM), Ottawa, ON, 2015, pp. 640-645.

[11] Dean DJ, Nguyen H, Gu X, "UBL: Unsupervised behavior learning for predicting performance anomalies in virtualized cloud systems", Proceedings of the 9th International Conference on Autonomic Computing (ICAC), San Jose, CA; 2012:191200.
[12] Khanum, Salma, and L. Girish. "Meta Heuristic Approach for Task Scheduling In Cloud Datacenter for Optimum Performance." International Journal of Advanced Research in Computer Engineering \& Technology (IJARCET) Volume 4.2015

[13] Nayana, Y., J. Gopinath, and L. Girish. "DDoS mitigation using Software Defined Network", International Journal of Engineering Trends and Technology (IJETT) 24.5 (2015): 258-264.

[14] Rashmi, T. V., and Keshava Prasanna. "Load Balancing As A Service In Openstack-Liberty." International Journal of Scientific \& Technology Research 4.8 (2015): 70-73.

[15] Influxdb: https://www.influxdata.com/

[16] collectd: https://collectd.org/

[17] stress-ng: http://kernel.ubuntu.com/ cking/stress-ng/ [18] iperf: https://iperf.fr/

[19] Marcel Wallschlger, Anton Gulenko, Florian Schmidt, Odej Kao, FengLiu, "Automated Anomaly Detection in Virtualized Services Using Deep Packet Inspection", Procedia Computer Science,Volume 110,2017,Pages 510515 ,

[20] L. Cao, P. Sharma, S. Fahmy, and V. Saxena, "Nfvvital: A framework for characterizing the performance of virtual network functions Network Function Virtualization and Software Defined Network", (NFVSDN),2015 IEEE Conference on, Nov 2015, pp. 9399.

[21] Miyazawa, M. and M. Hayashi. "In-network real-time performance monitoring with distributed event processing", IEEE Network Operations and Management Symposium. 2014. 